\title{
Dual isotope single-photon emission computerized tomography used for prediction of histology and survival in patients after high-dose radiotherapy for malignant astrocytoma
}

Richard B. Schwartz, M.D., Ph.D., B. Leonard Holman, M.D., Basem M. Garada, M.D., Paulo A. Carvalho, M.D., Rebecca Folkerth, M.D., Marc S. Schwartz, M.D., Jay S. Loeffler, M.D., Dennis C. Shrieve, M.D., Ph.D., Joseph F. Polak, M.D., Peter McL. Black, M.D., Ph.D., and Eben Alexander III, M.D.

Departments of Radiology, Pathology, Surgery, and Radiation Oncology, Brigham and Women's Hospital, Boston, Massachusetts

This study was conducted to determine the sensitivity of dual-isotope single-photon emission computerized tomography (SPECT) in predicting tumor recurrence and survival in patients treated with high-dose radiotherapy for malignant gliomas.

Studies using SPECT with thallium-201 (Tl-201) and technetium-99m (Tc-99m) hexamethypropyleneamine oxime (HMPAO) in 50 consecutive patients with malignant astrocytomas treated by surgery and high-dose radiotherapy were performed 1 day before reoperation. Maximum uptake of Tl-201 in the lesion was expressed as a ratio to that of the contralateral scalp, and uptake of Tc-99m HMPAO was expressed as a ratio to that of the cerebellar cortex. Patients were stratified into groups based on maximum radioisotope uptake values in their tumor beds. Differences in tumor histopathology at reoperation and 1-year survival between SPECT groups were determined by using chi-square analysis.

The majority of patients in Group IA (Tl-201 ratio less than 2 or Tc-99m HMPAO ratio less than 0.5) showed radiation changes in their biopsy specimens (85.7\%); they had an 85.7\% 1-year survival rate. Group II (Tl-201 ratio between 2 and 3.5) had predominantly infiltrating tumor (78.6\%); they had a $42 \%$ 1-year survival rate. Almost all of the patients in Group III (Tl-201 ratio greater than 3.5) had solid tumor (93.3\%) and they had a 6.7\% 1-year survival. Survival and pathological data differed significantly among groups (p less than 0.009).

Dual-isotope SPECT data correlates with histological findings on reoperation and postoperative survival in patients with malignant gliomas.

Key Words * glioblastoma * anaplastic astrocytoma * radionuclide imaging * radiation effects * recurrence * survival analysis

The present treatment for most patients with malignant astrocytomas (anaplastic astrocytoma (AA) or glioblastoma multiforme (GBM)) consists of tumor debulking followed by local-field external-beam 
radiation therapy. Many patients then receive stereotactic radiation boost therapy using either radiation implants [20,34] or radiosurgery,[28] which have been shown to be effective in increasing survival time in selected patients. $[8,17,19,20,28,30,34]$ Although a few patients are rendered free of progressive disease for several years by this therapy, the majority will eventually experience tumor recurrence, usually in the margins of the treatment field.[10,13] However, high-dose (greater than $60 \mathrm{~Gy}$ ) radiotherapy often produces exuberant gliosis and edema in the months following treatment that is clinically indistinguishable from recurrent tumor growth by standard radiographic means. $[3,9,10,13,33]$ Biopsy may be of limited diagnostic value because of sampling error.[4]

Functional imaging with thallium-201 (Tl-201) single-photon emission computerized tomography (SPECT) has recently been reported to be useful in predicting the presence and location of recurrent brain tumor after debulking and radiation treatment.[1,15,16,21,22,29,35] This technique has been found to be equal in accuracy to fluorine-18 deoxyglucose positron emission tomography for this purpose.[1,15] The radioisotope Tl-201 is a potassium analog that requires a disrupted blood-brain barrier (BBB) to gain access to the brain and is then taken up in proportion to the activity of the sodium-potassium adenosine triphosphatase pump. Previous reports that analyzed survival as a function of Tl-201 uptake in small populations did not analyze either the pattern of recurrence or the original tumor grade.[18,32] There have been no previous reports correlating survival time with findings on dual-isotope SPECT using Tl-201 and Tc-99m hexamethylpropyleneamine oxime (HMPAO). It has been shown that dual-isotope SPECT using Tc-99m HMPAO, a brain perfusion agent that crosses the intact $\mathrm{BBB}$, is more accurate than Tl-201 alone in discriminating radiation changes from recurrence.[5,27,36] The present report correlates the results of dual-isotope SPECT scanning with survival times and histopathological findings in a large population of patients treated with radiotherapy for either an AA or a GBM.

\section{CLINICAL MATERIAL AND METHODS}

\section{Patient Population}

Fifty consecutive patients, ranging in age from 21 to 75 years (mean 46.5 years), with supratentorial high-grade astrocytomas were included in our analysis. Thirty-eight patients were initially diagnosed as having a GBM and 12 were initially diagnosed with an AA. Some of these patients were included in a preliminary report from this institution.[5]

All patients were treated initially by bulk tumor resection, conventional radiotherapy (1.8 Gy daily, 5 days/week, for a total dose of $59.4 \mathrm{~Gy}$ ), and high-dose local radiotherapy, consisting of either iodine-125 radiation implants (12 patients) or stereotactic radiosurgery using a 6-MV linear accelerator (38 patients). Implants (brachytherapy) produced an approximately 50 Gy minimum dose over a 5-day period, and radiosurgery produced a 10 to 20 Gy dose in a single fraction. These two dose schemes provided similar tumoricidal effects within the treatment volume.[20] Only patients who experienced worsening of symptoms 3 months after high-dose local radiotherapy were analyzed; no patient was imaged with SPECT before the 3 month postoperative period to allow any immediate effects to resolve. Computerized tomography and/or magnetic resonance imaging in each patient showed postoperative changes in the region of the tumor bed and an irregular mass with heterogeneous peripheral enhancement and a hypocellular fluidic matrix centrally. Both the size of the mass and the degree of surrounding edema had increased in each patient since the time scans were obtained after the initial postoperative period. Dual-isotope SPECT was performed within 24 hours of the CT or magnetic resonance images. 


\section{Imaging Protocol}

After receiving an intravenous injection of $3 \mathrm{mCi}(111 \mathrm{MBq})$ of thallium chloride, all patients underwent imaging with a dedicated high-resolution brain SPECT system (CERASPECT brain imager; Digital Scintigraphics, Cambridge MA), which uses a single stationary annular sodium iodide crystal with an inside diameter of $31 \mathrm{~cm}$ and a thickness of $8 \mathrm{~cm}$. Data acquisition began 5 to 10 minutes after tracer injection and continued for 30 minutes, collecting 120 projections with a $360^{\circ}$ rotation of the collimators and using an energy window of $77 \pm 15 \mathrm{keV}$. After completion of data acquisition, and without moving the patient, 20 to $30 \mathrm{mCi}(740-1100 \mathrm{MBq})$ of Tc99m-HMPAO was injected intravenously. Data acquisition began 5 minutes after injection and continued for 30 minutes using dual energy windows $(140 \pm 14 \mathrm{keV}$ and $119 \pm 7 \mathrm{keV})$. Projections were processed and reconstructed using techniques detailed previously.[5,27]

The axial tomograms were displayed on a $128 \mathrm{X} 128$ matrix (1.67-sq $\mathrm{mm}$ pixel size) as a set of 64 slices (1.76-mm slice thickness). These slices were summed to give a final display of 21 slices, with a slice thickness of $5 \mathrm{~mm}$. The spatial resolution was $7.8 \mathrm{~mm}$ (full width at one-half the maximum count) for Tl-201 and $7.4 \mathrm{~mm}$ for Tc-99m at $5 \mathrm{~cm}$ from the center. The Tl-201 axial tomograms were displayed using a color scale that identified the picture elements with the highest activity in the dataset $(75 \%$ $100 \%$ of the maximum slice activity). The axial slice with the highest count density in the known tumor area was chosen for quantitative analysis. An elliptical region of interest (ROI) was drawn manually to encompass the area of the brightest pixels. A second polygonal ROI with a fixed width of two pixels (3.3 $\mathrm{mm}$ ) was drawn over the contralateral scalp. A Tl-201 tumor/scalp activity ratio was obtained from the ratio of the average counts per pixel in these two ROIs. The Tc-99m HMPAO tumor/cerebellum ratio was determined from the average counts per pixel in an ROI copied from the corresponding Tl-201 tomogram and a polygonal ROI with a fixed 2-mm pixel width drawn over the cerebellar cortex (Fig. 1).

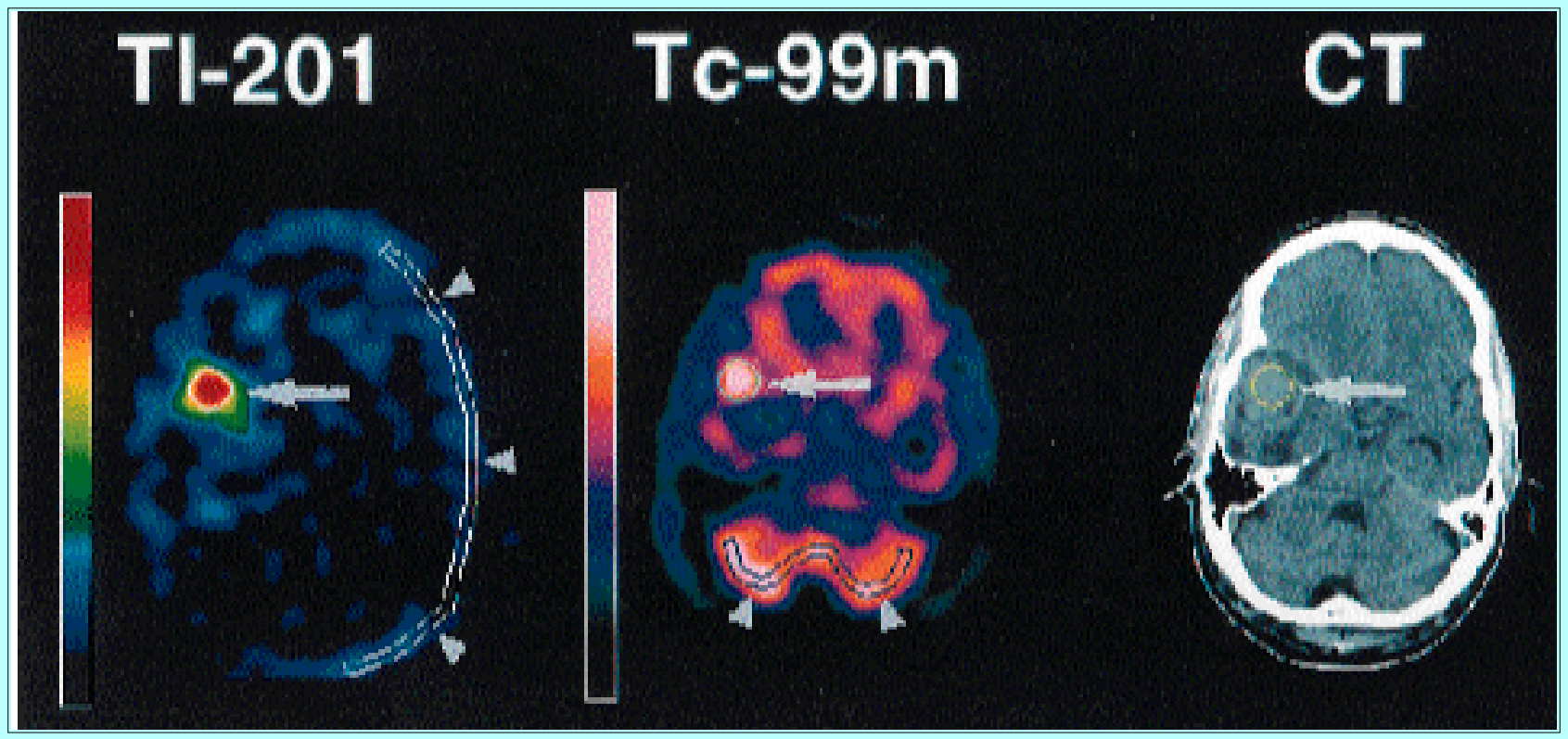

Fig. 1. Illustration of the method for semiquantification of single-photon emission computerized tomography (SPECT) data. Left: Axial thallium-201 (Tl-201) scan through a treated tumor bed showing a circular region of interest (ROI) surrounding the site of highest intracranial Tl-201 uptake (arrow) that is used to correlate with technetium-99m (Tc-99m) hexamethylpropyleneamine oxime (HMPAO) uptake. A 2-mm thick ROI band (arrowheads) encompassing the contralateral scalp is used as an internal standard to define a ratio of 
uptake. Center: Axial Tc-99m HMPAO scan through the same level as the Tl-201 scan showing the ROI superimposed (arrow). An axial scan through the mid-cerebellum showing a 2-mm thick ROI band (arrowheads) including the cerebellar cortex at this level, which is used as an internal standard to define a ratio of uptake. Right: Axial CT scan obtained after the administration of intravenous contrast material through the same level as the SPECT scans showing the ROI superimposed on the scan, within an area of nonspecific heterogeneous enhancement (arrow).

\section{Surgical Procedure and Pathological Diagnosis}

In each case, maximal excision was performed so as to include all regions of abnormal Tl-201 uptake, and it was extended to include as much abnormally enhancing tissue as possible. Intraoperative motor and speech mapping was used when necessary, and in those few patients in whom eloquent cortex was identified within the surgical field, resection was accomplished to within 10 to $15 \mathrm{~mm}$ of its margins. Patients were followed for at least 1 year after the SPECT examinations or until the time of death.

Stereotactic biopsies were obtained using the Cosman-Roberts-Wells stereotactic apparatus (Radionics Inc., Burlington MA), based on image fusion between the SPECT and stereotactic CT scans.[14] All biopsies were obtained through individual burrholes before mannitol and furosemide administration to allow precise correlation of imaging and biopsy points without distortion of brain tissue. Complete resection of the necrotic tumor mass was then accomplished through a formal craniotomy. En bloc excisions were oriented at the time of operation. Tissues were fixed in $10 \%$ formalin and routinely paraffin embedded, cut, and stained with hematoxylin and eosin. Analysis of the stereotactic specimens was performed in such a manner as to allow exact correlation of the spatial coordinates of SPECT, CT, and magnetic resonance images with histopathological findings.

Histological evaluation of excised and biopsy tissue was performed with attention to several parameters. [7,12] Sparse dysplastic astrocytes in a matrix of gliosis and necrotic tissue were believed to represent radiation changes (Fig. 2 upper). The growth pattern of the recurrent or residual astrocytoma, when present, was recorded as either infiltrating tumor (that is, cells infiltrating intact brain parenchyma; Fig. 2 center), or solid tumor (that is, cells displacing brain parenchyma, Fig. 2 lower). Histological evaluation was performed without knowledge of the results of preoperative SPECT scanning.

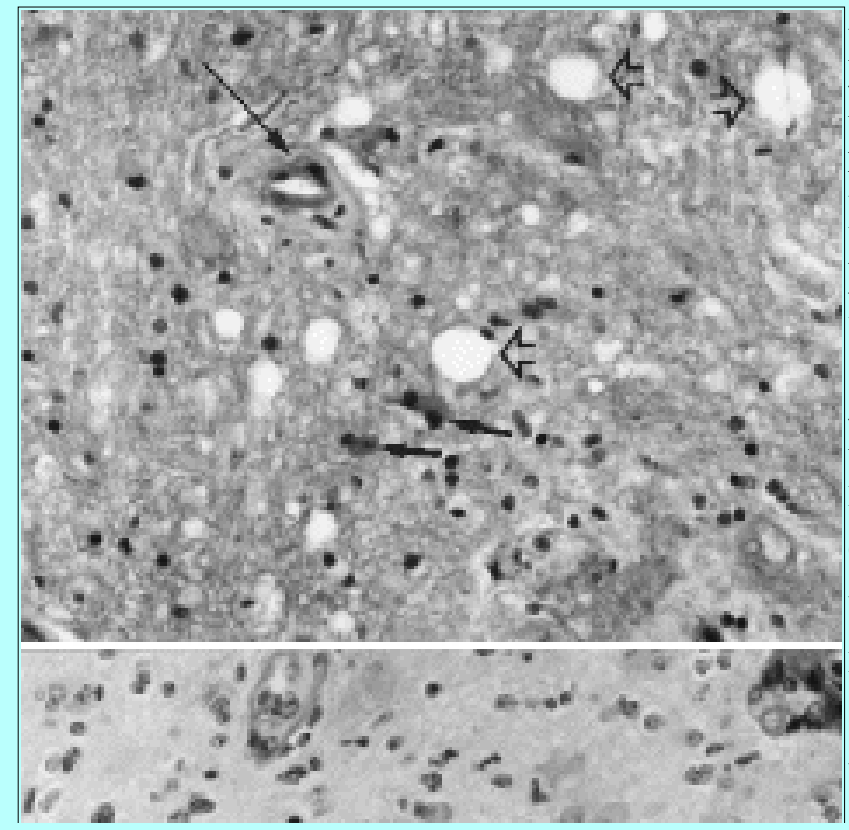

Fig. 2. Photomicrographs showing representative histopathological findings at reoperation following radiotherapy of malignant astrocytomas. Upper: Photomicrograph revealing radiation necrosis. Reactive posttreatment changes in cerebral white matter include vascular wall thickening (long arrow) and reactive astrocytosis (short arrows). The field is relatively hypocellular. No tumor cells are evident. Vacuoles in intact parenchyma (open arrows) indicate interstitial edema. Center: Photomicrograph revealing infiltrating tumor. Infiltrating tumor cells (long arrows) are readily identified by their irregular nuclear outlines (pleomorphism), hyperchromatism, and their tendency to cluster near neurons (satellitosis, at center of field). A 


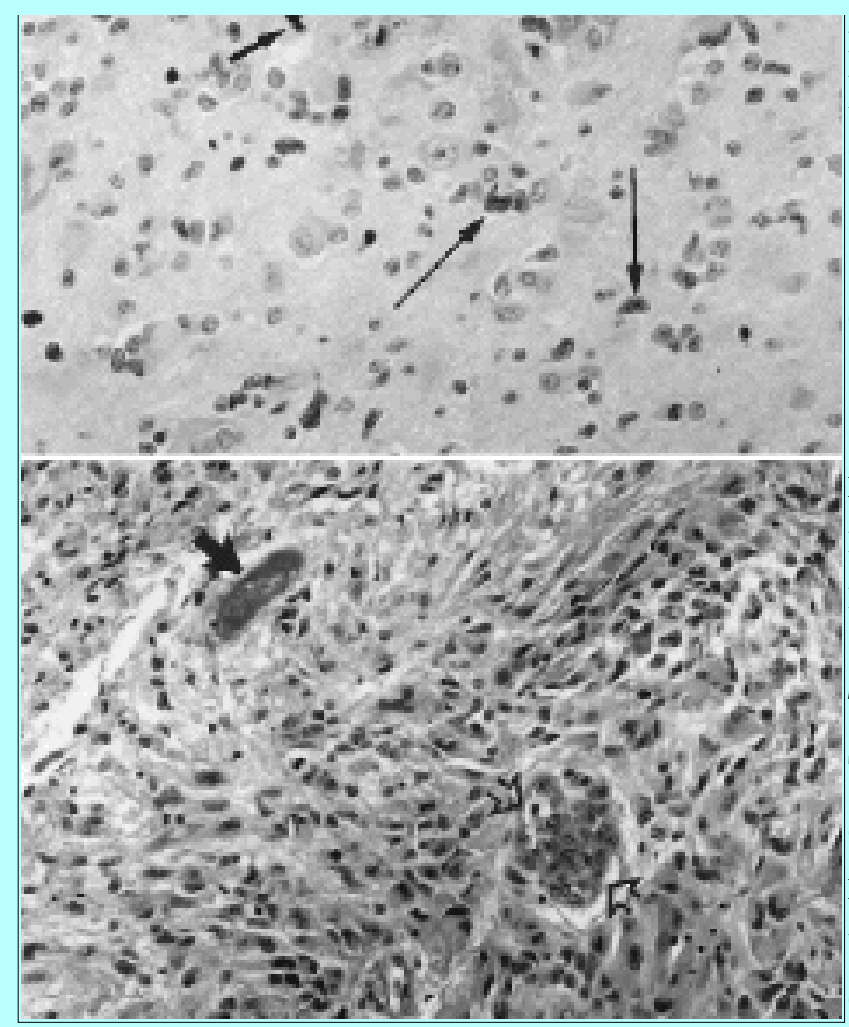

mitotic figure (short arrow) is demonstrated. Lower: Photomicrograph revealing solid tumor. This is a densely cellular tumor with sheets of abnormal astrocytes shown in the lower right of the field. A "glomeruloid" vessel (that is, with multilayered endothelial proliferation) is present in this portion of the specimen (open arrows). Necrosis and macrophage accumulation is present in the upper left of the field; radiation-induced fibrinoid change is present in the necrotic area (closed arrow). $\mathrm{H} \& \mathrm{E}$, original magnifications X 1000.

\section{Statistical Analysis}

The reference point for this study was the date of the SPECT examination. Time from initial diagnosis to date of radiotherapy was determined, as was the time from treatment to SPECT study; the time from SPECT investigation to the endpoint (date of death or January 1, 1996) was also calculated. The main outcome was survival at 1 year following the SPECT examination. The Student's t-test was used to evaluate the significance of differences between the mean values of continuous variables (age and time from diagnosis to SPECT). The chi-square statistic was used to determine differences between dichotomous variables (namely gender, original tumor grade, and survival at 1 year) or ordinal data (histological data at reoperation) for SPECT uptake groups. The Fisher exact chi-square test was used when the events per cell were five or less. It should also be noted that because only single Tl-201 data points were analyzed, no attempt was made to correlate survival with tumor size or volume of resection.

Further analysis of the predictive power of SPECT data on mortality was performed using the methods of Kaplan and Meier. Survival curves were stratified by the calculated SPECT ratios. Differences were evaluated by the log-rank method. The possible effects due to confounding variables were controlled for by a Cox proportional hazards model that included as independent variables SPECT grouping, age, gender, and tumor grade. Significance was determined at the .05 level.

\section{RESULTS}

\section{Single-Photon Emission Computerized Tomography Analysis}

Low Tl-201 Uptake (ratio less than 2): Group I. There was no significant difference in the survival times of all patients with low Tl-201 uptake from that of patients with higher Tl-201 ratios. However, Tc-99m HMPAO values differentiated the patients with necrosis (those who showed low Tc-99m HMPAO uptake) from those with recurrent tumor (most of whom showed high Tc-99m HMPAO uptake, $p$ less than 0.001).

Low Tc-99m HMPAO: Group IA. Seven patients had Tl-201 ratios less than 2 and Tc-99m HMPAO ratios less than 0.5. Six patients in this group had GBMs, and one had an AA. The patient with the AA had infiltrating tumor on biopsy. Six patients $(85.7 \%)$ showed a radiation change only on biopsy, without evidence of active tumor growth. Six patients $(85.7 \%)$ in this group were alive after 1 year, including the 
patient diagnosed with infiltrating tumor. One patient died from sepsis and had no evidence of recurrent tumor at autopsy. Patients in the low Tl-201 and low Tc-99m HMPAO group had significantly better survival times at 1 year than all other patients (p less than 0.05, Fig. 3A).

High Tc-99m HMPAO: Group IB. All four patients who had Tl-201 ratios less than 2 and Tc-99m HMPAO ratios greater than or equal to 0.5 had GBMs, and infiltrating tumor was noted on biopsy in each one (100\%). None of these patients was alive at 1 year; one died from pneumonia and the others from tumor recurrence. The time to recurrence in these patients was approximately one-third of that in the other patients (4.25 months vs. 12.7 months); this difference did not reach statistical significance. These patients had significantly worse survival times than those in Group IA ( $p$ less than 0.025).

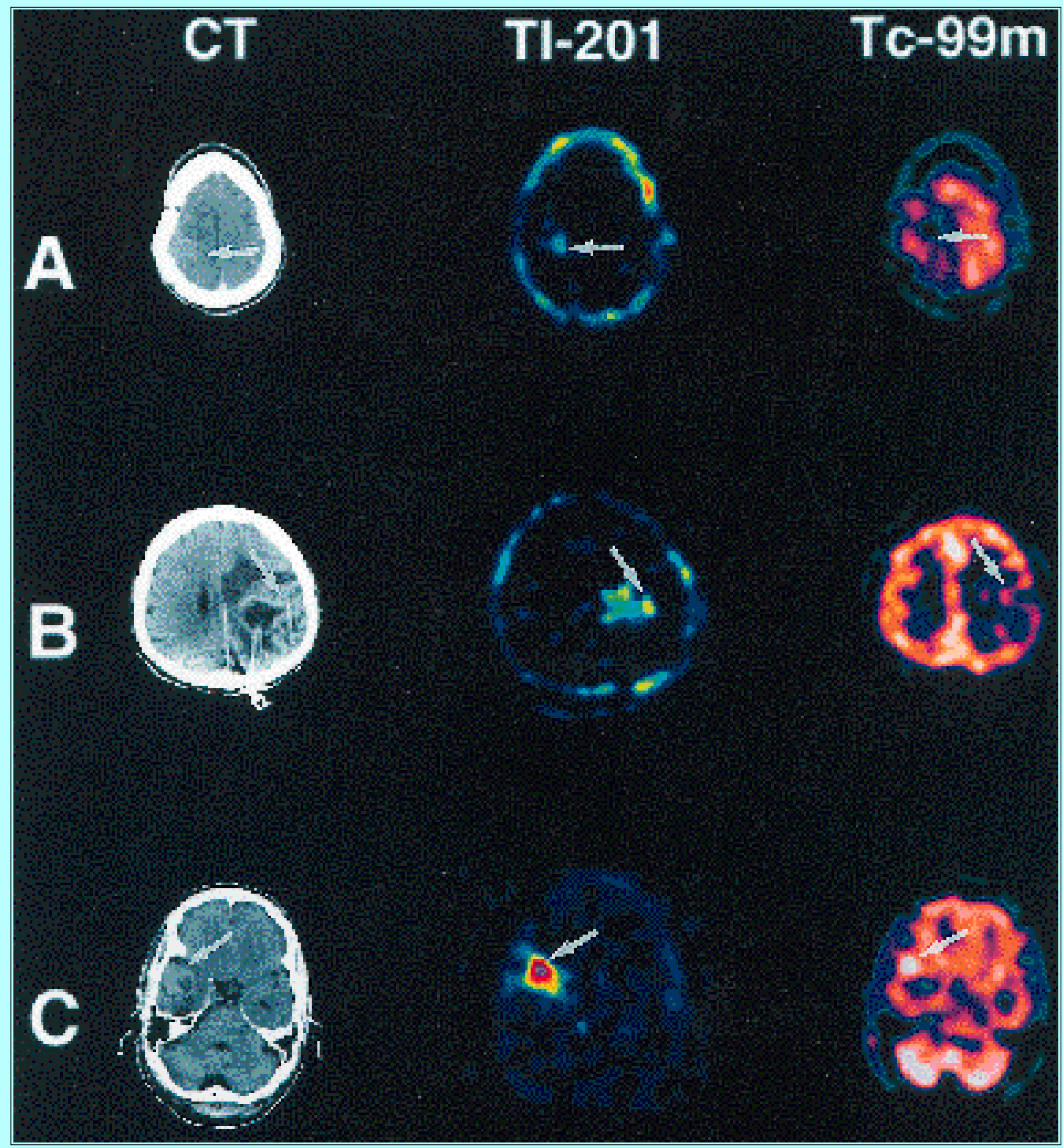

Fig. 3. Stereotactic computerized tomography (CT), thallium-201 (Tl-201), and technetium-99m (Tc-99m) hexamethylpropyleneamine oxime (HMPAO) scans obtained through the same axial level in patients from Groups IA, II, and III, respectively. In each case, arrows on the scans indicate the region within the treatment bed that corresponds to the site of maximal Tl-201 uptake (center). Note that the CT scans in each case show nonspecific postoperative changes with varying amounts of enhancement and necrosis. A: Scans in a patient from Group I. Thallium-201 lesion/scalp uptake ratio is low (less than 2) and the Tc-99m HMPAO lesion/cerebellum uptake ratio is low (less than 0.5); this patient showed benign radiation changes on biopsy. B: Scans in a patient from Group II. 
Thallium-201 lesion/scalp uptake ratio is moderate (greater than 2, and less than 3.5), and the Tc-99m HMPAO lesion/cerebellum uptake ratio is low (less than 0.5); this patient had infiltrating tumor on biopsy. C: Scans in a patient from Group III. Thallium-201 lesion/scalp uptake ratio is high (greater than 3.5) and Tc-99m HMPAO lesion/cerebellum uptake ratio is high (greater than 0.5 ); this patient had solid tumor on biopsy.

Uptake of Tl-201 Between 2 and 3.5: Group II. Low Tc-99m HMPAO: Group IIA. Group IIA consisted of 14 patients who had Tl-201 ratios between 2 and 3.5 and Tc-99m HMPAO ratios less than or equal to 0.5. Five patients had AAs and nine had GBMs. Of these patients, only one (7.8\%) showed radiation changes on biopsy; this patient died within 5 months. The remainder of these patients had active tumor on biopsy; 11 (78.6\%) of these patients had infiltrating tumor, and two (15.4\%) had solid tumor on biopsy. Seven patients $(50 \%)$ in this group were alive at 1 year; one died from a pulmonary embolus and the others from recurrent tumor (Fig. 3B).

High Tc-99m HMPAO: Group IIB. Group IIB consisted of 10 patients (four with AAs and six with GBMs), none of whom had benign radiation changes. Six patients $(60 \%)$ had infiltrating tumor, and four $(40 \%)$ had solid tumor on biopsy. Four $(40 \%)$ of the patients in this group were alive at 1 year. There was no significant difference in the pathological findings or the survival times in patients in Groups IIA and IIB.

Uptake of Tl-201 Greater than 3.5: Group III. Of 15 patients with Tl-201 ratios greater than or equal to 3.5, all had Tc-99m HMPAO ratios greater than or equal to 0.5, and $14(93.3 \%)$ had recurrence in the form of solid tumor; the remaining patient with infiltrating tumor had an AA and was the only patient alive 12 months after SPECT examination. All others in this group had GBMs and were dead 1 year after SPECT studies. The survival time of patients in this group was significantly worse than the remainder of the patients (p less than 0.05; Fig. 3C).

\section{Intergroup Comparisons}

For purposes of comparison, patients were stratified into three major groups: Group IA, Group II, and Group III (Table 1). There were too few Group IB patients to be considered as a separate group for statistical analysis; however, they were clearly different from patients in Group IA, and because there was no a priori reason to include those patients with any other group, they were excluded from further intergroup analysis. Patients in Groups IIA and IIB did not differ significantly in terms of pathological findings or survival time and so were grouped together for intergroup comparisons. 


\begin{tabular}{|c|c|c|c|}
\hline \multicolumn{4}{|c|}{$\begin{array}{c}\text { TABLE } 1 \\
\text { MAJOR SURYMAL GROUPS }\end{array}$} \\
\hline \multirow[b]{2}{*}{ Characteristic } & \multicolumn{3}{|c|}{ Group } \\
\hline & IA & II & III \\
\hline \multicolumn{4}{|l|}{ patient data } \\
\hline no. of patients & 7 & 24 & 15 \\
\hline no. with AAS & 1 & 10 & 1 \\
\hline no. with GBMs & 6 & 14 & 14 \\
\hline pathology results & & & \\
\hline no. with necrosis & 6 & 1 & 0 \\
\hline no. with infiltrating tumor & 1 & 17 & 1 \\
\hline no. with solid tumor & 0 & 6 & 14 \\
\hline $1-y r$ surwival rate by & & & \\
\hline group (no. of patients) & $85.7 \%(6 /)$ & $45.8 \%(11 / 24)$ & $6.7 \%(1 / 15)$ \\
\hline
\end{tabular}

Histological Findings. Uptake in the three major groups was predictive of histological findings on reoperation. Significantly more patients in Group IA showed radiation changes only in their biopsy specimens $(85.7 \%)$ than in Group II (4.2\%; p less than 0.001). Significantly more patients in Group II had infiltrating tumor on biopsy (70.8\%) than in either Group IA (14.3\%; p less than 0.01$)$ or Group III (6.7\%; p less than 0.001$)$, and significantly more patients in Group III had solid tumor $(93.3 \%)$ than did those Group II (25\%; p less than 0.001). There was no significant difference in the age of the patients in the three groups.

Survival Times. Patients in Group IA had significantly better survival times than those in Group II ( $85.7 \%$ vs. $41.7 \%$ alive at 1 year, p less than 0.05 ). Patients in Group II had better survival times than those in Group III ( $41.7 \%$ vs. $6.7 \%$ alive at 1 year, p less than 0.025$)$. As Fig. 4 illustrates, the survival rates of these three groups were significantly different ( $\mathrm{p}$ less than 0.0009), and survival rates were worse for those with higher SPECT scores. It should be noted that including the Group IB patients with either Groups II or III did not appreciably alter the results of the histopathological or survival analysis. The Cox proportional hazards model showed that the relative risk of mortality was strongly affected by the SPECT score. After controlling for variables such as tumor grade, gender, and age, only the SPECT score predicted survival time ( $\mathrm{p}$ less than 0.005). 


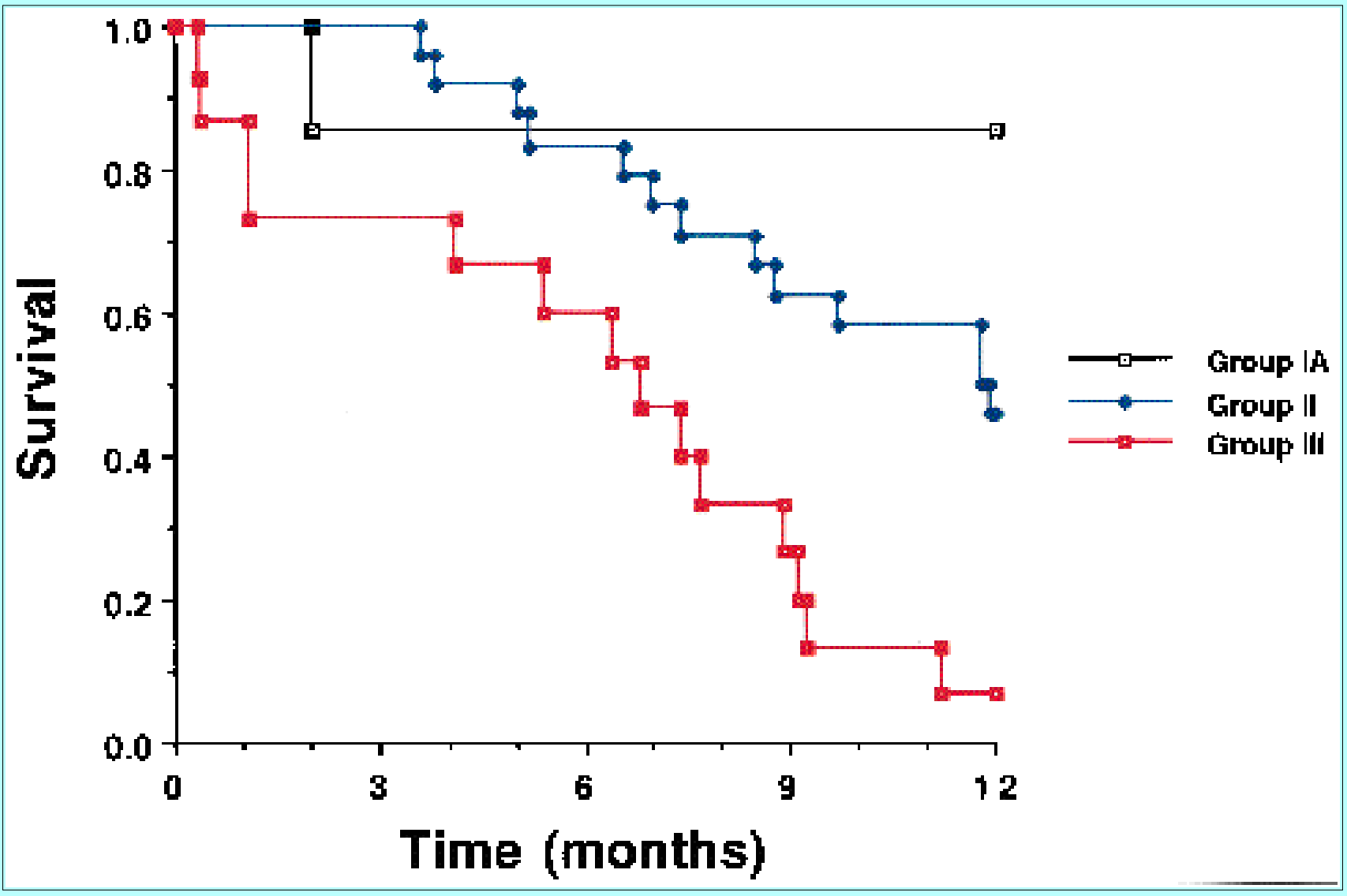

Fig. 4. Graph displaying survival curves of the three major single-photon emission computerized tomography uptake groups. The black line (open squares) represents Group IA patients; the blue line (closed circles) represents patients in Group II; and the red line (closed squares) represents patients in Group III. The three curves are significantly different from each other ( $\mathrm{p}$ less than 0.009).

\section{Original Tumor Grade}

The mean age of the 38 patients with GBMs $(50.2 \pm 13.8$ years) was significantly greater than that of the 12 patients with AAs $(34.8 \pm 8.3$ years; $p$ less than 0.001$)$. The time from treatment to the presumed recurrence was $9.86 \pm 10.0$ months for patients with the initial diagnosis of GBM, significantly shorter than that for patients with AA $(18.7 \pm 12.0$ months; $p$ less than 0.025$)$. One patient with an AA showed radiation changes on biopsy, and all other patients with AAs had infiltrating tumor. Six patients with GBM showed radiation changes on biopsy, 11 of the 38 patients with GBM had infiltrating tumor, and the remaining 21 had solid tumor. Patients with AAs had longer survival times than those with GBM; of the 12 patients with AAs, nine (66.7\%) were alive at 1 year, whereas $11(29.7 \%)$ of the 37 patients with GBMs were alive at 1 year ( $\mathrm{p}$ less than 0.05). There was also an apparent effect of gender on original tumor type in our patients; $10(83.3 \%)$ of the 12 patients with AAs were women, whereas $31(81.6 \%)$ of those with GBMs were men ( $\mathrm{p}$ less than 0.05). The importance, if any, of this association is unclear.

The SPECT uptake groups correlated with original tumor grade. Patients with AAs were more likely to be in Group II, and patients with GBMs to be in Group III ( $p$ less than 0.05). However, the difference in survival times between Group II and Group III was independent of original tumor grade. Among the patients with GBMs, those in Group II had a significantly better survival time than those with GBM in Group III (p less than 0.01). 


\section{Comparison of Isotope Uptake}

Technetium-99m HMPAO levels were predictive of survival; patients with low Tc-99m HMPAO uptake had significantly better survival times than did those with high Tc-99m HMPAO (p less than 0.01). Uptake of Tl-201 and Tc-99m HMPAO was also correlated $(r=0.58 ; p$ less than 0.005).

The efficacy of results obtained for single- and dual-isotope SPECT examinations in the determination of recurrent tumor were as follows. For single-isotope SPECT using Tl-201 alone and a cut-off of 2, the sensitivity was $88.4 \%$, the specificity was $85.7 \%$, the negative predictive value was $54.5 \%$, and the positive predictive value was $97.4 \%$. The sensitivity of Tc-99m HMPAO scans alone using a cut-off of 0.5 was $67.4 \%$, the specificity was $100 \%$, the negative predictive value was $33.3 \%$, and the positive predictive value was 100\%. For dual-isotope SPECT (Tl-201 cut-off of 2 and Tc-99m HMPAO cut-off of 0.5 ), the sensitivity was $97.4 \%$ and the specificity was $85.7 \%$, resulting in an accuracy of $98.0 \%$. The negative predictive value was $85.7 \%$ and the positive predictive value was $98.0 \%$.

\section{DISCUSSION}

The survival of patients after therapy for malignant gliomas has been correlated with various factors including original tumor diagnosis and patient age [6], but few investigators have assessed the utility of SPECT data for predicting survival after treatment. Kosuda, et al.[18] and Vertosik, et al.[32] showed an inverse relationship between Tl-201 uptake at reoperation and survival time; however, those authors examined relatively small groups of glioma patients and only a single Tl-201 cut-off was used, limiting the applicability of the results to a larger population of patients. The present investigation expands these earlier analyses by establishing more detailed correlations of SPECT data with histopathological findings and patient survival time in a large homogeneous population of patients with high-grade astrocytomas. In agreement with preliminary results in previous studies,[5,27] we found that all patients with Tl-201 lesion/scalp ratios greater than 3.5 had high Tc-99m HMPAO uptake (greater than 0.5 that of the cerebellum) as well; these patients (Group III) tended to have highly cellular tumor recurrences on biopsy and had worse survival times at 1 year than all other patients. We further defined a subgroup of patients with Tl-201 uptake ratios between 2 and 3.5 (Group II), which was composed predominantly of patients with less cellular tumor recurrences and with better 1-year survival times $(42 \%)$ than the patients in Group III (7\% 1-year survival); in Groups II and III, Tc-99m HMPAO uptake did not contribute significantly to the results of the analysis.

In patients with Tl-201 uptake ratios less than 2 (Group I), Tc-99m HMPAO uptake predicted both the histopathological findings and survival at 1 year. Only those patients in Group I who also had low Tc-99m HMPAO uptake showed benign radiation changes in their biopsies at reoperation (Group IA), and they had an excellent 1-year survival time (85.7\%); all of those in Group I with high Tc-99m HMPAO uptake had recurrent infiltrating tumor on biopsy (Group IB) and were dead within 1 year. There was a significant difference in survival times between Groups IA and IB; indeed, patients in Group IA had significantly better survival times at 1 year than all other patients. The benefit of Tc-99m HMPAO SPECT examination in the setting of low Tl-201 uptake may relate to differences in the mechanism of uptake of the two isotopes. Thallium-201 uptake requires a disrupted BBB to gain entry into the brain parenchyma, whereas Tc-99m HMPAO does not. Based on the short time for recurrence and poor outcome of the patients in Group IB, it is possible that at the time of SPECT scanning, tumor was infiltrating rapidly through brain tissue. Under these circumstances, perfusion (and therefore Tc- $99 \mathrm{~m}$ HMPAO uptake) may have increased due to angiogenesis $[11,25]$ prior to significant BBB disruption. If 
so, early tumor regrowth in such cases may be signaled by increased Tc-99m HMPAO uptake before Tl-201 uptake is perceptibly altered. Further consideration of these points would require serial SPECT scans with pathological correlation.

Although we found that high Tc-99m HMPAO uptake predicts recurrent tumor and poor survival and also correlates with Tl-201 uptake, we do not recommend the use of Tc-99m HMPAO alone for the determination of recurrent tumor. Low Tc-99m HMPAO uptake may occur in the presence of recurrent tumor (and increased Tl-201) if radiation damage to the surrounding normal brain interferes with angiogenesis (that is, Group IIA).[25] More important, islands of normal brain within a treatment field may be mistaken for recurrent tumor if Tl-201 is not used to ensure that these are associated with BBB breakdown. Specifically, although SPECT scanning using Tc-99m HMPAO alone is associated with a very high specificity and positive predictive value in the evaluation of recurrent tumor, it has a sensitivity of $67 \%$ and a negative predictive value of only $33 \%$. Thus, in the setting of possible tumor recurrence, Tc-99m HMPAO scanning is best performed as part of a dual-isotope SPECT study. Indeed, our data indicate that dual-isotope SPECT using both Tl-201 and Tc-99m HMPAO determines recurrent tumor after radiotherapy with greater accuracy than is possible with SPECT scanning using either isotope alone.

We found that SPECT scanning also correlated with initial histological appearance of the tumor. Almost all of the patients with AAs were in Group II; and in agreement with other authors, $[8,19]$ we found that patients with AAs had less aggressive disease both in terms of the rapidity of regrowth after radiotherapy and the cellularity of the recurrence than did patients with GBMs. However, even among the patients with GBM, significantly more in Group II were alive at 1 year than in Group III. Indeed, the correlation of Tl-201 uptake with survival time was independent of tumor type, age, or gender.

The ability of SPECT scanning to predict long-term survival before reoperation deserves further comment. All patients were initially treated with bulk resection followed by radiotherapy of sufficient dosage to control tumor within the field;[26] thus, regions of high Tl-201 uptake on SPECT scanning obtained several months after radiotherapy most likely represented the continued growth of tumor cells that were just outside the center of the radiation field (marginal failures). Although repeat surgery was then performed to remove as much pathological tissue as possible, including the regions of abnormal Tl-201 uptake, in most cases recurrences occurred; furthermore, the activity of the tumor cells (as inferred from survival times) was predicted by the preoperative Tl-201 uptake in the region of the tumor bed. This implies that the recurrences represented centripetal growth of tumor cells that had already spread beyond the margins of grossly abnormal tissue at the time of the reoperation.[2,31]

Accurate determination of death rates resulting from tumor growth in patients with malignant astrocytomas is inherently difficult because of the generally debilitated status of these individuals after therapy. Several patients in our series who did not experience recurrence died from such conditions as pneumonia, sepsis, and pulmonary embolism. In other cases, fatal systemic illnesses intervened in patients with known recurrence, whereas in others, autopsies were not performed so the cause of death could not be specifically determined. For purposes of survival analysis, however, all fatalities were considered to be equivalent. This may have resulted in a conservative bias in the low-uptake group and an overestimation of the death rate due to recurrence in the high-uptake group. Similarly, although histological data at the time of reoperation were used as the standard to determine the efficacy of SPECT scanning, in fact, pathological diagnosis can be problematic in the setting of radiation effects. It may be particularly difficult to distinguish reactive astrocytosis from infiltrating neoplastic glial cells in these patients.[3] In the present study, the one patient with the false-positive SPECT result died from recurrent 
tumor 5 months after biopsy, and the patient with the false-negative study remains alive 20 months after biopsy. These findings suggest that SPECT studies may have been more accurate than histological findings in predicting the proliferative capacity of the abnormal astrocytes in these cases. Recently developed immunohistochemical methods such as bromodeoxyuridine uptake analysis are likely to be more effective than standard histological studies for determining the behavior of astrocytes in the tumor bed;[24] a recent study has shown a positive correlation between bromodeoxyuridine and Tl-201 uptake.[23]

\section{CONCLUSIONS}

We conclude that dual-isotope SPECT scanning, in addition to effectively localizing sites of recurrent astrocytoma following high-dose radiotherapy, accurately predicts the histological appearance of tumor at reoperation and, more important, long-term survival of these patients. This information could be used to guide further therapy; in some circumstances, SPECT studies may obviate the need for repeat biopsy in the posttreatment period. Future studies may include the use of serial SPECT scanning obtained at intervals before and after treatment to help elucidate further the spatiotemporal patterns of growth of high-grade astrocytomas.

\section{References}

1. Black KL, Emerick T, Hoh C, et al: Thallium-201 SPECT and positron emission tomography equal predictors of glioma grade and recurrence. Neurol Res 16:93-96, 1994

2. Burger PC: Malignant astrocytic neoplasms: classification, pathologic anatomy, and response to treatment. Semin Oncol 13:16-26, 1986

3. Burger PC, Dubois PJ, Schold SC Jr, et al: Computerized tomographic and pathologic studies of the untreated, quiescent, and recurrent glioblastoma multiforme. J Neurosurg 58:159-169, 1983

4. Burger PC, Kleihues P: Cytologic composition of the untreated glioblastoma with implications for evaluation of needle biopsies. Cancer 63:2014-2023, 1989

5. Carvalho PA, Schwartz RB, Alexander E III, et al: Detection of recurrent gliomas with qualitative thallium-201/technetium-99m HMPAO single-photon emission computerized tomography. J Neurosurg 77:565-570, 1992

6. Curran WJ Jr, Scott CB, Horton J, et al: Recursive partitioning analysis of prognostic factors in three Radiation Therapy Oncology Group malignant glioma trials. J Nat Cancer Inst 85:704-710, 1993

7. Daumas-Duport C, Scheithauer BW, Kelly PJ: A histologic and cytologic method for the spatial definition of gliomas. Mayo Clin Proc 62:435-449, 1987

8. Devaux BC, O'Fallon JR, Kelly PJ: Resection, biopsy, and survival in malignant glial neoplasms. J Neurosurg 78:767-775, 1993

9. Dooms GC, Hecht S, Brant-Zawadzki M, et al: Brain radiation lesions: MR imaging. Radiology 158:149-155, 1986

10. Duncan GG, Goodman GB, Ludgate CM, et al: The treatment of adult supratentorial high grade astrocytomas. J Neurooncol 13:63-72, 1992 
11. Folkman J, Merler E, Abernathy C, et al: Isolation of a tumor factor responsible for angiogenesis. J Exp Med 133:275-288, 1971

12. Giangaspero F, Burger PC: Correlations between cytologic composition and biologic behavior in the glioblastoma multiforme. A postmortem study of 50 cases. Cancer 52:2320-2333, 1983

13. Halperin EC, Bentel G, Heinz ER, et al: Radiation therapy treatment planning in supratentorial glioblastoma multiforme: an analysis based on post mortem topographic anatomy with CT correlations. Int J Radiat Oncol Biol Phys 17:1347-1350, 1989

14. Holman BL, Zimmerman RE, Johnson KA, et al: Computer-assisted superimposition of magnetic resonance and high-resolution technetium-99m-HMPAO and thallium-201 SPECT images of the brain. J Nucl Med 32:1478-1484, 1991

15. Kahn D, Follett KA, Bushnell DL, et al: Diagnosis of recurrent brain tumor: value of 201Tl SPECT vs 18F-fluorodeoxyglucose PET. AJR 163:1459-1465, 1994

16. Kim KT, Black KL, Marciano D, et al: Thallium-201 SPECT imaging of brain tumors: methods and results. J Nucl Med 31:965-969, 1990

17. Kitchen ND, Hughes SW, Taub NA, et al: Survival following interstitial brachytherapy for recurrent malignant glioma. J Neurooncol 18:33-39, 1993

18. Kosuda S, Fujii H, Aoki S, et al: Prediction of survival in patients with suspected recurrent cerebral tumors by quantitative thallium-201 single photon emission computed tomography. Int J Radiat Oncol Biol Phys 30:1201-1206, 1994

19. Leibel SA, Scott CB, Loeffler JS: Contemporary approaches to the treatment of malignant gliomas with radiation therapy. Semin Oncol 21:198-219, 1994

20. Loeffler JS, Alexander E III, Shea WM, et al: Radiosurgery as part of the initial management of patients with malignant gliomas. J Clin Oncol 10:1379-1385, 1992

21. Mountz JM, Stafford-Shuck K, McKeever PE, et al: Thallium-201 tumor/cardiac ratio estimation of residual astrocytoma. J Neurosurg 68:705-709, 1988

22. Moustafa HM, Omar WM, Ezzat I, et al: 201Tl single photon emission tomography in the evaluation of residual and recurrent astrocytoma. Nucl Med Commun 15:140-143, 1994

23. Oriuchi N, Tamura M, Shibazaki T, et al: Clinical evaluation of thallium-201 SPECT in supratentorial gliomas: relationship to histologic grade, prognosis and proliferative activities. J Nucl Med 34:2085-2089, 1993

24. Ritter AM, Sawaya R, Hess KR, et al: Prognostic significance of bromodeoxyuridine labeling in primary and recurrent glioblastoma multiforme. Neurosurgery 35:192-198, 1994

25. Schiffer D, Giordana MT, Sofietti R, et al: Histological observations on the regrowth of malignant gliomas after radiotherapy and chemotherapy. Acta Neuropathol 58:291-299, 1982

26. Schwartz M, Loeffler JS, Black PM, et al: Reoperation following radiosurgery of glioblastoma: impact on survival and neurologic status. Radiosurgery (In press, 1996) 
27. Schwartz RB, Carvalho PA, Alexander E III, et al: Radiation necrosis vs high-grade recurrent glioma: differentiation by using dual-isotope SPECT with 201Tl and 99mTc-HMPAO. AJNR 12:1187-1192, 1991

28. Shrieve DC, Alexander E III, Wen PY, et al: Comparison of stereotactic radiosurgery and brachytherapy in the treatment of recurrent glioblastoma multiforme. Neurosurgery 36:275-282, 1995

29. Slizofski WJ, Krishna L, Katsetos CD: Thallium imaging for brain tumors with results measured by a semiquantitative index and correlated with histopathology. Cancer 74:3190-3197, 1994

30. Stea B, Rossman K, Kittelson J, et al: Interstitial irradiation versus interstitial thermoradiotherapy for supratentorial malignant gliomas: a comparative survival analysis. Int J Oncol Biol Phys 30:591-600, 1994

31. Tracqui P, Cruywagen GC, Woodward DE, et al: A mathematical model of glioma growth: the effect of chemotherapy on spatio-temporal growth. Cell Prolif 28:17-31, 1995

32. Vertosick FT Jr, Selker RG, Grossman SJ, et al: Correlation of thallium-201 single photon emission computed tomography and survival after treatment failure in patients with glioblastoma multiforme.

Neurosurgery 34:396-401, 1994

33. Wallner KE, Galicich JH, Malkin MG, et al: Inability of computed tomography appearance of recurrent malignant astrocytoma astrocytoma to predict survival following reoperation. J Clin Oncol 7:1492-1496, 1989

34. Wen PY, Alexander E III, Black PM, et al: Long term results of stereotactic brachytherapy used in the initial treatment of patients with glioblastomas. Cancer 73:3029-3036, 1994

35. Yoshii Y, Satou M, Yamamoto T, et al: The role of thallium-201 single photon emission tomography in the investigation and characterization of brain tumours in man and their response to treatment. J Nucl Med 20:39-45, 1993

36. Zhang JJ, Park CH, Kim SM, et al: Dual isotope SPECT in the evaluation of recurrent brain tumor. Clin Nucl Med 17:663-664, 1992

Manuscript received July 23, 1996.

Accepted in final form August 16,1996.

Address reprint requests to: Richard B. Schwartz, M.D. Ph.D., Division of Neuroradiology, Brigham and Women's Hospital, 75 Francis Street, Boston, Massachusetts 02115.

Click here to go to Editor's Perspective on 1. 